\title{
Review: in people with bipolar disorder, short term antidepressants improve clinical response, although tricyclics risk inducing mania
}

Gijsman HJ, Geddes JR, Rendell JM, et al. Antidepressants for bipolar depression: A systematic review of randomized, controlled trials. Am J Psychiatry 2004;161:1537-47.

\section{What are the clinical effects of antidepressants in short term therapy of depressive episodes in people with bipolar disorder?}

\section{METHODS}

$\square$

Design: Systematic review.

Data sources: Cochrane Collaboration Depression, Anxiety, and Neurosis Controlled Trials Register (December 2002), MEDLINE

(1966 to present), EMBASE (1980 to present), CINAHL (1982 to present), PsycLIT (1974 to present), PSYNDEX (1977 to present), and LILACS (1982-1999) plus hand search of reference lists, papers, and textbooks covering affective disorder.

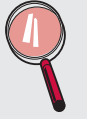

Study selection and analysis: Inclusion criteria: double blind randomised controlled trial (RCT) comparing antidepressants with placebo or alternative drug treatments; published in any language; people with a current depressive or mixed depressive/ manic episode with or without psychotic symptoms and at least one previous episode of mania or hypomania. Analysis: pooled relative risk calculated for binary efficacy outcomes using fixed and random effects models. Heterogeneity was assessed using the $Q$ statistic.

Fine mania.

\section{MAIN RESULTS}

Twelve RCTs met inclusion criteria $(\mathrm{n}=1088)$.

Antidepressants versus placebo: antidepressants significantly increased clinical response and remission compared with placebo, and there was no significant difference in risk of switching to mania (response: $\mathrm{n}=662$, RR $1.86,95 \%$ CI 1.49 to 2.30 ; remission: $\mathrm{n}=573$, RR 1.41, 95\% CI 1.11 to 1.80 ; switching: $n=779$, RR $1.00,95 \%$ CI 0.47 to 2.13$)$.

Tricyclic antidepressants versus other antidepressants: there was no significant difference in clinical response and remission between tricyclic antidepressants and other antidepressants, although tricyclic antidepressants significantly increased switching to mania compared with other antidepressants (response: $\mathrm{n}=296$, RR $0.84,95 \%$ CI 0.67 to 1.06 ; remission: $\mathrm{n}=74$, $\mathrm{RR} 0.84,95 \%$ CI 0.47 to 1.48 ; switching: $\mathrm{n}=370$, RR $2.92,95 \%$ CI 1.28 to 6.71 ).

\section{CONCLUSIONS}

In people with bipolar disorder, antidepressants increase clinical response and remission compared with placebo, and do not increase the risk of switching to mania. Tricyclic antidepressants increase the risk of switching to mania compared with other antidepressants. There is limited evidence on the effects of antidepressants versus other medications. Further studies are needed that assess longer follow up periods and clearly define emerging mania and partial remission.

\section{NOTES}

Most people in the RCTs were also taking a mood stabiliser or an atypical antipsychotic, so the results may not be applicable to antidepressant monotherapy.

For correspondence: Harm Gijsman, Scutari Clinic, St Thomas' Hospital Lambeth Palace Road, London, UK; harm.giijsman@doctors.net.uk

Sources of funding: none reported.

\section{Commentary}

7 his systematic review and meta-analysis by Gijsman et al provides an important new perspective on the reluctance to prescribe antidepressants, especially the newer ones, for bipolar depression-a difficult-to-treat condition.

However, the analyses are limited by the paucity and short duration of studies, the absence of a placebo arm in several studies, the concomitant use of a mood stabiliser in the majority of participants, and the lack of detail regarding the history of rapid cycling or antidepressant induced mania. The studies are also made heterogeneous by inclusion of people with bipolar II depression, mixed episodes, and a history of only antidepressant induced mania. We must await further studies in order to be confident about the low rate of switching from depression to mania with antidepressants in the absence of a concomitant mood stabiliser. Antidepressants may not be as efficacious for bipolar depression when added to a mood stabiliser at the higher end of the therapeutic range. For example, no difference was found in response between paroxetine and placebo when added to lithium at levels $>0.8 \mathrm{mEq} / \mathrm{l}$.

When deciding whether to prescribe an antidepressant, potential alternatives should be considered which have little risk of promoting a switch, such as lamotrigine, ${ }^{2}$ lithium, quetiapine, ${ }^{3}$ or olanzapinefluoxetine combination. ${ }^{4}$ The use of olanzapine (and perhaps quetiapine) must, however, take into account the risk of metabolic syndrome. Lamotrigine and lithium also have the advantage of being efficacious for prophylaxis of bipolar disorder.

There are data to suggest a very low rate of switching with paroxetine ${ }^{1}$ and bupropion. ${ }^{5} \mathrm{~A}$ small study suggested that there may be a lower rate of switching with paroxetine than with venlafaxine, ${ }^{6}$ but this finding needs to be replicated. Hence we believe the American Psychiatric Association guideline regarding the choice of antidepressant is supported by some evidence, even though it is limited.

Maximising the mood stabiliser and/or the addition of lamotrigine or lithium is currently recommended as the first step in treatment for bipolar depression. If an antidepressant is needed, using medication such as paroxetine or bupropion, for which at least some data exist to support a very low rate of switch, is clinically reasonable. However, use of antidepressants without a concomitant mood stabiliser is not recommended. Further data are needed to clarify the role of second generation antipsychotics as adjunctive treatments for bipolar depression.

Prakash Masand, MD Duke University Medical Center, Durham, NC, USA Rajnish Mago, MD Thomas Jefferson University, Philadelphia, PA, USA

1 Nemeroff CB, Evans DL, Gyulai L, et al. Double-blind, placebo-controlled comparison of imipramine and paroxetine in the treatment of bipolar depression. Am J Psychiatry 2001;158:906-12.

2 Calabrese JR, Bowden CL, Sachs GS, et al. A double-blind placebocontrolled study of lamotrigine monotherapy in outpatients with bipolar I depression. Lamictal 602 Study Group. J Clin Psychiatry 1999;60:79-88.

3 Calabrese J, et al. Presented at the 157th Annual Meeting of the American Psychiatric Association, New York, 2004.

4 Tohen $M$, et al. Presented at the 157th Annual Meeting of the American Psychiatric Association, New York, 2004.

5 Sachs GS, Lafer B, Stoll AL, et al. A double-blind trial of bupropion versus desipramine for bipolar depression. J Clin Psychiatry 1994;55:391-3.

6 Vieta E, Martinez-Aran A, Goikolea JM, et al. A randomized trial comparing paroxetine and venlafaxine in the treatment of bipolar depressed patients taking mood stabilizers. J Clin Psychiatry 2002;63:50812. 